\title{
Event-based proactive interference in rhesus monkeys
}

\author{
Deepna T. Devkar ${ }^{1}$ • Anthony A. Wright ${ }^{1}$
}

Published online: 9 February 2016

(C) Psychonomic Society, Inc. 2016

\begin{abstract}
Three rhesus monkeys (Macaca mulatta) were tested in a same/different memory task for proactive interference (PI) from prior trials. PI occurs when a previous sample stimulus appears as a test stimulus on a later trial, does not match the current sample stimulus, and the wrong response "same" is made. Trial-unique pictures (scenes, objects, animals, etc.) were used on most trials, except on trials where the test stimulus matched potentially interfering sample stimulus from a prior trial $(1,2,4,8$, or 16 trials prior). Greater interference occurred when fewer trials separated interference and test. PI functions showed a continuum of interference. Delays between sample and test stimuli and intertrial intervals were manipulated to test how PI might vary as a function of elapsed time. Contrary to a similar study with pigeons, these time manipulations had no discernable effect on the monkey's PI, as shown by compete overlap of PI functions with no statistical differences or interactions. These results suggested that interference was strictly based upon the number of intervening events (trials with other pictures) without regard to elapsed time. The monkeys' apparent event-based interference was further supported by retesting with a novel set of 1,024 pictures. PI from novel pictures 1 or 2 trials prior was greater than from familiar pictures, a familiar set of 1,024 pictures. Moreover, when potentially interfering novel stimuli were 16 trials prior, performance accuracy was actually greater than
\end{abstract}

Deepna T. Devkar

deepna.85@gmail.com

Anthony A. Wright

anthony.a.wright@uth.tmc.edu

1 Department of Neurobiology \& Anatomy, The University of Texas Medical School, Houston, TX 77030, USA accuracy on baseline trials (no interference), suggesting that remembering stimuli from 16 trials prior was a cue that this stimulus was not the sample stimulus on the current trial-a somewhat surprising conclusion particularly given monkeys.

Keywords Proactive interference - Visual working memory · Same/different $\cdot$ Long-term memory $\cdot$ Monkeys

Picture memory or memory for photographic stimuli of animals, objects, and scenes can last for days, or seemingly be forgotten in a few seconds. For example, humans have been shown to remember over 2,000 images (90\% retention) even after 3 days of delay between learning and testing of 2,560 picture stimuli (Standing, Conezio, \& Haber, 1970). In contrast, rhesus monkeys have been shown to forget a single picture after a mere 30-s delay (Overman \& Doty, 1980). Surely, monkeys can remember things like multicolored pictures for more than 30 seconds! In that particular experiment, there were a small number of picture stimuli that were repeated many times within a session. In a second experiment, these researchers tested the same monkeys with a larger picture set of 100 familiar pictures, presented trial-unique within sessions. The monkeys performed substantially better, and even better $(65 \%)$ after a $24-\mathrm{hr}$ delay than they had with a $30-\mathrm{s}$ delay with the smaller repeated set of stimuli. Moreover, in a third experiment these researchers obtained even better performance ( $80 \%$ after a 24 -hr delay) when the picture stimuli were novel and presented trial-unique across the testing sessions. These improvements in memory raise the question of why memory performance is so poor with a small, repeated stimulus set. The answer lies in the well-documented phenomenon of proactive interference (PI) across many animal and human memory studies (Cowan, 2005; Hartshorne, 2008; Jonides \& Nee, 2006; Makovski \& Jiang, 2008; Wright, 
Urcuioli, \& Sands, 1986). Keppel and Underwood (1962) showed in a verbal memory task, that PI cumulatively builds up over trials as the same stimuli are repeated in the same memory testing session, leading to PI saturation and confusion about which to-be-remembered stimuli were seen on the current trial as opposed to previous trials.

By using a large set of stimuli (sampled without replacement), the background level of PI was shown to be minimized and performance by a rhesus monkey in a list-memory task dramatically improved (Sands \& Wright, 1980). With such a minimized background level of PI, one can then test how far back in time PI can interfere with memory performance on the current trial. In one such study, the extent of PI was systematically tested by placing potentially interfering items at different points prior to the current trial in a 10-item list-memory paradigm (Wright, Urcuioli, \& Sands, 1986). When the interfering items were in the immediately preceding trial, performance was $64 \%$ (chance performance was $50 \%$ ). As the separation between the interfering and target item increased (up to as many as 60 items between), performance increased to $83 \%$. Nevertheless, this performance was still substantially less accurate than the no-PI baseline performance (93\% correct). This systematic approach provides better control and manipulation of PI, allowing a better understanding of the functional relationship of PI and memory performance over a continuum of interference.

The purpose of the present experiment was to study PI in rhesus monkeys by using trial-unique stimuli within sessions to reduce the background level of PI and to systematically manipulate controlled tests of PI to determine the extent to which PI affects the monkeys' memory performance. We modeled our task after a similar study with pigeons that tested PI with single stimulus items in a same/different procedure (Wright, Katz, \& Ma, 2012; also, see Fig. 5 in the "General Discussion"). In this pigeon study, a large stimulus set of 1,024 images (pictures of objects, natural scenes, animals, etc.) was used so that stimuli would be trialunique and not repeated for at least 2 weeks of testing. PI was tested by placing potentially interfering stimuli at $1,2,4,8$, and 16 trials prior to test trials. With a $1-\mathrm{s}$ delay between the sample and test of each trial, interfering stimuli in the immediately preceding trial produced a $17 \%$ PI effect, which increased to $42 \%$ when the delay was increased to 10 seconds. These delay differences in the magnitude of the PI effect were shown to obey a Weber-Fechner time ratio: time at test to current-trial sample stimulus divided by time to interfering stimulus (cf., Buhusi \& Meck, 2005; Gibbon, 1977). Here, we investigated whether the PI function for monkeys would show a time relationship similar to pigeons or some other relationship, for example, PI based on events rather than time.

\section{Experiment 1}

Experiment 1 tested the monkey's PI at three delays: $1 \mathrm{~s}, 10 \mathrm{~s}$, and $20 \mathrm{~s}$, to substantially alter the time ratio between the current and interfering stimuli.

\section{Method}

\section{Subjects}

Three adult male rhesus monkeys (Macaca mulatta; weights: 14.1-17.5 kg; ages: $10.5-15.5$ years at the beginning of the experiment) were tested in a same/different task. Testing was conducted for 5 to 7 days a week. Food and water were restricted prior to experimental sessions. After completing daily testing, the monkeys received a standard diet of primate chow and water in their colony room, where they were housed individually. On days that the monkeys were not tested, they were given supplemental enrichment (fruits and vegetables) in addition to the daily diet to maintain their normal body weight. All animal procedures conformed to the National Institutes of Health guidelines, and were approved by the Institutional Review Board as well as the Institutional Animal Care and Use Committee at University of Texas Health Science Center at Houston.

\section{Apparatus}

The monkeys were trained and tested unrestrained in a custom-made aluminum experimental chamber $(47.5 \mathrm{~cm}$ wide $\times 53.1 \mathrm{~cm}$ deep $\times 66.3 \mathrm{~cm}$ high). The touch responses were detected by an infrared touchscreen that was attached to a 17" computer monitor (NEC, Multisync, $800 \times 560$ resolution). A Plexiglas template with three rectangular cutouts matching the size and location of two vertically aligned travel slide stimuli (each $11.5 \mathrm{~cm} \times 9.3 \mathrm{~cm}$ ) separated by a $1.4-\mathrm{cm}$ gap between them, and a white rectangle $(6.2 \mathrm{~cm} \times 5.6 \mathrm{~cm})$ located to the right of the lower picture to guide the touch responses (see Fig. 1a). Experimental sessions were designed, operated, and recorded using a custom program written in Microsoft Visual Basic 6.0. Correct responses were rewarded with either a banana pellet or orange-flavored Tang juice using a computercontrolled relay interface (Model P10-12; Metrabyte, Taunton, MA). Throughout testing, the monkeys were monitored with a video camera outside the chamber and focused through a small glass covered port on the right side of the chamber.

\section{Stimuli}

Stimuli consisted of colored images of objects, natural scenes and animals that were displayed on a black background. Based on the average distance of the monkey from the screen 
a

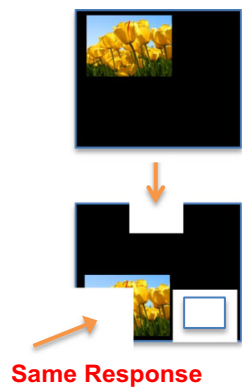

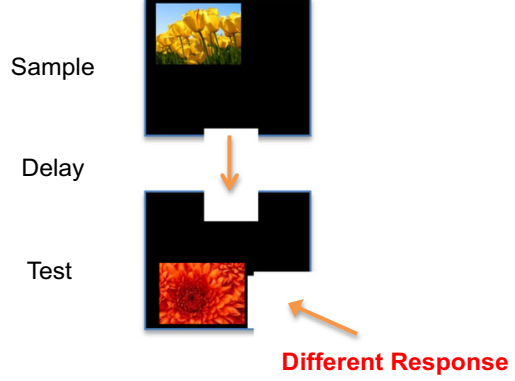

b

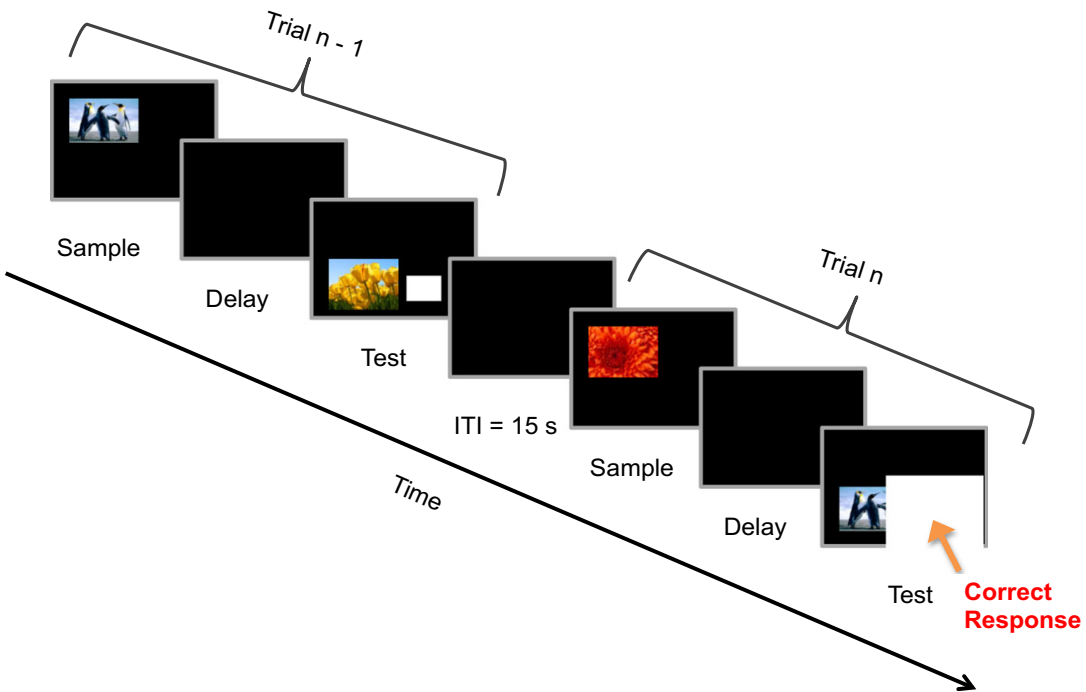

Fig. 1 a Same/Different task procedure shown (but not to scale) with examples of same (left) and different (right) trials. The monkey makes a one-touch response to the sample stimulus (bunch of tulip flowers), which is presented on the top left panel of the screen. Followed by a delay (either $1 \mathrm{~s}, 10 \mathrm{~s}$, or $20 \mathrm{~s}$, depending on the testing condition), the test stimulus (either tulips or dahlia) is presented (bottom left) on the screen with a white rectangle (bottom right). If the sample and test stimuli are identical, then the correct response is to touch the test stimulus, indicating a "same"

(approximately $30 \mathrm{~cm}$ ), the stimuli subtended a visual angle of $7.22^{\circ} \mathrm{h} \times 10.76^{\circ} \mathrm{w}$. On every trial, the sample stimulus was presented on the top and the test stimulus was presented on the bottom simultaneously with a white rectangle, which appeared to the right of the test stimulus. The bottom of the white rectangle (the different response area) was horizontally aligned with the bottom of the lower picture (the same response area) and separated by a $3.7-\mathrm{cm}$ gap.

\section{Procedure}

Each trial began with an auditory tone that was played continuously until the monkeys made one touch response to the location where the sample stimulus would be displayed. The touch response initiated the presentation of the sample stimulus (shown at the top left of examples of same and different trials in Fig. 1a.). The monkeys were required to touch the sample stimulus, which was randomly chosen from a set of 1,024 travel response. If the same and test stimuli are not identical, then the correct response is to touch the white rectangle, indicating a "different" response. b An example of a PI test showing two trials, where the test stimulus (three penguins) that differs from the sample stimulus on the current trial, $n$, was seen as a sample stimulus on the preceding trial, $n-1$. Thus, PI could potentially produce confusion as to whether the test stimulus matches the sample stimulus on the current trial or the sample stimulus seen on the previous trial slides. The sample stimulus disappeared upon touch, and then after a delay ( $1 \mathrm{~s}, 10 \mathrm{~s}$, or $20 \mathrm{~s})$, the test stimulus was presented (bottom left) with a white rectangle (bottom right). If the two stimuli were the same, then the correct response was to touch the test stimulus; if the images were different, the correct response was to touch the white rectangle next to the test stimulus (see Fig. 1b). The test stimulus and the white rectangle remained on the screen until response. Correct responses were rewarded with either orange-flavored Tang juice or a 1-gm banana pellet (selected pseudorandomly). An intertrial interval of $15 \mathrm{~s}$ followed choice responses, during which a green light (located outside the chamber and above a gap between the touch screen and computer monitor) illuminated the chamber and the otherwise dark screen.

The time between the offset of the sample stimulus and the onset of the test stimulus (delay) was manipulated to examine the effect of time on PI as shown in Table 1. Each of the three monkeys was tested at three different delays $(1 \mathrm{~s}, 10 \mathrm{~s}$, and 
Table 1 Time (seconds) Between the Interfering Stimulus and the Test Stimulus

\begin{tabular}{|c|c|c|c|c|c|}
\hline & \multicolumn{5}{|c|}{ Interference Location in Trials Prior to Test $(n)$} \\
\hline & $n-1$ & $n-2$ & $n-4$ & $n-8$ & $n-16$ \\
\hline \multicolumn{6}{|c|}{ Experiment 1 (Delay) } \\
\hline $1 \mathrm{~s}$ & 19.4 & 37.7 & 74.4 & 147.8 & 294.6 \\
\hline $10 \mathrm{~s}$ & 38.1 & 65.4 & 120.1 & 229.5 & 448.3 \\
\hline $20 \mathrm{~s}$ & 58.1 & 95.4 & 170.1 & 319.5 & 618.3 \\
\hline \multicolumn{6}{|c|}{ Experiment 2 (ITI) } \\
\hline $5 \mathrm{~s}$ & 48.1 & 75.4 & 130.1 & 239.5 & 458.3 \\
\hline $15 \mathrm{~s}$ & 58.1 & 95.4 & 170.1 & 319.5 & 618.3 \\
\hline
\end{tabular}

20 s) in six-session blocks at each delay. Each session consisted of 84 trials (50\% were same trials and $50 \%$ were different trials), 74 baseline trials and 10 interference test trials. Baseline trials were composed of randomly selected pictures from the 1,024 items set (without replacement). This selection procedure resulted in two weeks of testing before any stimuli were repeated on baseline trials. Interference test trials were always different trials and consisted of potentially interfering stimuli placed $1,2,4,8$, or 16 trials prior to the test. There were two tests of each of the five separations quasirandomly placed in each session. Figure $1 \mathrm{~b}$ shows an example of two trials, where the test stimulus that differs from the sample stimulus on the current trial, $n$, was seen as a sample stimulus on the preceding trial, $n-1$. Thus, PI could potentially produce confusion as to whether the test stimulus matches the sample stimulus on the current trial or a stimulus that was previously seen on another trial.

\section{Training and testing}

All three monkeys had been trained and tested on a procedure similar to the same/different procedure used here with the same 1,024 stimulus set for over five years. At the beginning of the present experiment, the monkeys were retrained with trial-unique baseline trials (50\% same and $50 \%$ different trials; no tests of PI) in the same/different procedure for 12 to 24, 96-trial sessions to ensure adequate baseline performance. When each monkey performed at $85 \%$ correct or better on baseline trials, then testing PI began with a block of six sessions with a 1-s delay, then a block of six sessions with a 10-s delay, and final block of six sessions with a 20 -s delay. All other task parameters were held constant.

\section{Results and discussion}

Figure 2 shows the mean memory performance of three monkeys for five separations between the interfering stimulus and test compared with baseline performance (no-PI trials) across

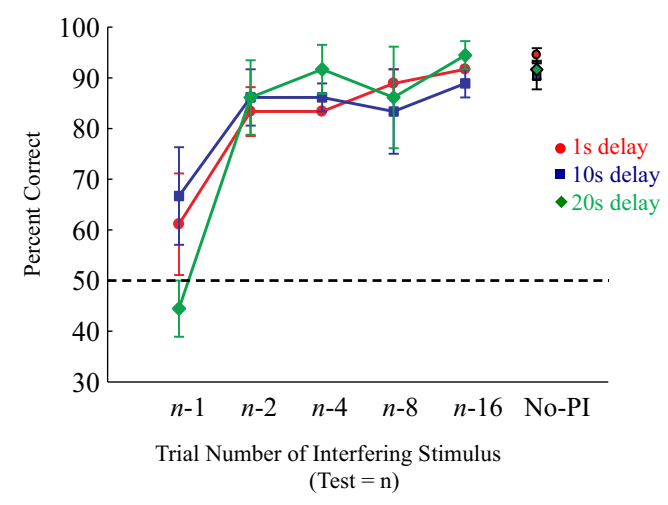

Fig. 2 Mean performance of three monkeys at five interfering-stimulus separations on interference trials (PI trials) compared with baseline performance (No-PI trials) for 1-s, 10-s, and 20-s delays. The intertrial interval was fixed at $15 \mathrm{~s}$ across all conditions. The error bars represent mean \pm standard error of the mean across monkeys

1-s, 10-s, and 20-s delays. A two-way repeated measures analysis of variance (ANOVA) of Delay $\times$ Intervening Items (between the interference trial and the test trial) showed a significant main effect of Intervening Items across 6 conditions: $n-1$, $n$-2, $n-4, n-8, n$-16, No-PI; $F(5,10)=20.41, p<.001$. At each delay condition, performance was least accurate at the $n-1$ condition, showing the greatest effect of interference; for example, at the 20-s delay, memory performance was below chance at $44.4 \%$ for the $n-1$ condition. The interaction of Delay $\times$ Intervening Items was not significant, $F(10,20)=$ $2.08, p=.079$. The main effect of delay also was not significant, $F(2,4)=0.051, p=.95$, showing that the amount of time separating the interfering and the test stimulus had little or no effect on performance accuracy. An ordinary least squares (OLS) multivariate regression analysis also showed that intervening items, but not delay, significantly predicted performance. For these analyses, delays ( $1 \mathrm{~s}, 10 \mathrm{~s}$, and $20 \mathrm{~s})$ and intervening items ( $n-1, n-2, n-4, n-8, n-16$, and No-PI) were treated as categorical predictors, compared to the No-PI condition (baseline condition), and explained $63.1 \%$ of the variance, $F(7,46)=11.22, p<.001$. The $n-1$ condition significantly predicted performance ( $\beta=-34.5, t=-7.45, p<.001$ ), but delay did not (all $p \mathrm{~s}>.70$ ).

This lack of an effect of delay is contrary to findings from pigeons, despite large time differences across these delay conditions as shown in Table 1. Table 1 shows the total time between the offset of the interfering stimulus to the onset of the test stimulus, and also includes average response times for the intervening trials. For example, in the $n-1$ condition and a 1-s delay, the time between the offset of the interfering stimulus and the test stimulus includes: the delay period from the interfering trial $(1,000 \mathrm{~ms})$, the response time (average of M1 $=1,650 \mathrm{~ms})$, the intertrial interval $(15 \mathrm{~s})$, the touch response time for the current trial's sample stimulus (mean $=700 \mathrm{~ms}$ ), and the delay period to the test stimulus ( $1,000 \mathrm{~ms})$. Adding all of these together, the elapsed time would be on average $19.4 \mathrm{~s}$. In the same $n-1$ condition, when the delay is $10 \mathrm{~s}$, the 
elapsed time increases to $38.1 \mathrm{~s}$ and about $58.1 \mathrm{~s}$ when the elapsed time is 20 -s. As the number of trials between the interfering stimulus and the test stimulus is increased, the difference in elapsed time across delays multiplies accordingly; for example, for $n-4$, the elapsed time for the 1-s delay would be $74.4 \mathrm{~s}$, whereas that for the 10-s delay would be $120.1 \mathrm{~s}$. Although delay did increase with intervening trials, the considerable 20 fold increase in delay had no discernable effect ( $p$ $=.95$ ) on PI; which is even more remarkable due to increased discrimination difficulty from the longer delay on the PI test trial. Indeed, if one looks at the condition where there are no intervening trials $(n-1)$ to assess whether delay had any functionality, then the $58.1 \mathrm{~s}$ for the 20 -s test should have produced much less PI than under the 1-s condition (19.4-s delay). But it did not. If anything just the reverse was true. And also PI from the 20 -s delay test in the $n$ - 1 condition $(58.1 \mathrm{~s})$ should have been less than PI from the $n-2$ condition $(37.7 \mathrm{~s}$ ) for the $1-\mathrm{s}$ delay test. But here too that clearly was not the case. The longest time in this experiment was more than 10 minutes (n-16, 20-s delay) and was more than a two-fold increase over the longest time in the 1-s delay conditions. Nevertheless, all three PI functions overlapped one another showing no effect of this considerable time change. This lack of an effect of delay suggests that perhaps monkeys and pigeons have fundamental differences in how memory is processed and how memories interact and interfere with one another in terms of time separations and/or event separations.

\section{Experiment 2}

In Experiment 1, the delay between sample stimulus and test stimulus had little or no discernable effect on memory performance in monkeys, unlike for pigeons. Another way to manipulate time is to alter the intertrial interval (ITI). By substantially reducing the intertrial interval from $15 \mathrm{~s}$ to $5 \mathrm{~s}$ would move the potentially interfering stimuli considerable closer to the test stimulus, possibly increasing confusion about whether an interfering stimulus was or was not the sample stimulus on the test trial. This was the purpose of Experiment 2. We kept the delay time constant at $20 \mathrm{~s}$ so that the Weber-Fechner fraction (time at test to current-trial sample stimulus divided by time to interfering stimulus) would be highly skewed toward showing a relative time effect on PI.

\section{Method}

\section{Procedure}

In this experiment, we manipulated time by testing effects of PI at two ITIs: $5 \mathrm{~s}$ and $15 \mathrm{~s}$. Each monkey was first tested with six-session blocks of 15-s ITIs and then with six-session blocks of 5-s ITIs. Memory delays between the sample and test were kept constant at $20 \mathrm{~s}$ in both conditions. The rationale for using a 20-s delay for these ITI manipulations was that the monkeys had recently been well performing the task with this delay, and this longer delay should make memory performance more vulnerable to any changes in PI that might occur when interference was more recent (5-s ITIs) as opposed to more distant (15-s ITIs). All other aspects of the procedure were unchanged from Experiment 1.

\section{Results and discussion}

Figure 3 shows the mean performance of the three monkeys at the same six conditions ( $n-1, n-2, n-4, n-8, n-16$, No-PI) for 5 $\mathrm{s}$ and 15-s ITIs. In a two-way repeated measures analysis of variance $($ ANOVA) of ITI $\times$ Intervening items, we again found a significant main effect of intervening items across the six conditions: $n-1, n-2, n-4, n-8, n-16$, No-PI; $F(5,10)=$ $11.51, p=.001$. The interaction of ITI $\mathrm{x}$ Intervening items was not significant, $F(5,10)=0.45, p=0.081$. The main effect of ITI was also not significant, $F(1,2)=3.97, p=.19$. An ordinary least squares (OLS) multivariate regression analysis also showed that intervening items, but not delay, significantly predicted performance. For these analyses, ITI times ( $5 \mathrm{~s}$ and $15 \mathrm{~s})$ and intervening items ( $n-1, n-2, n-4, n-8, n-16$, and No_PI) were treated as categorical predictors, compared to the No-PI condition (baseline condition), and explained $77.6 \%$ of the variance in the data, $F(6,29)=16.78, p<.001$. The $n-1$ and $n-4$ significantly predicted performance $(n-1: \beta=-48.9, t=-7.84, p<.001 ; n-4$ : $\beta=-22.6, t=-3.62, p<.001$ ), but the ITI did not (all $p \mathrm{~s}>.50$ ).

As in Experiment 1, complete overlap of the PI functions, lack of a significant effect of ITI (time), and lack of a significant interaction suggests that the amount of time separating the interfering stimulus and the test stimulus had little or no effect on performance accuracy. These results, combined with our findings from Experiment 1, suggest that any mere

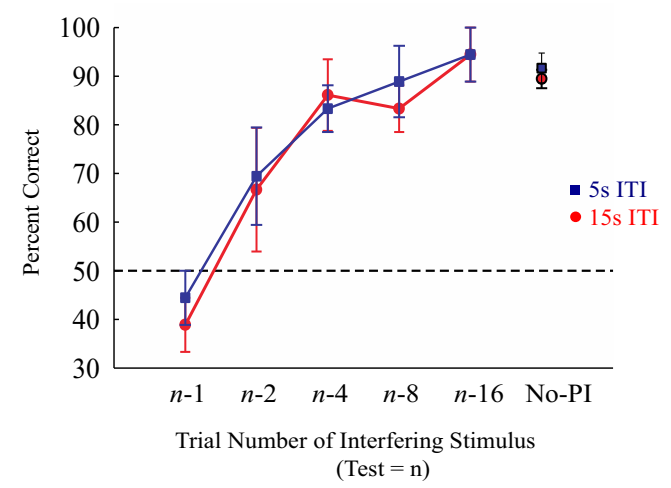

Fig. 3 Mean performance of three monkeys at five interfering-stimulus separations on interference trials (PI trials) compared with baseline performance (No-PI trials) across 5-s and 15-s intertrial intervals. The delay between the sample stimulus and test stimulus was fixed at $20 \mathrm{~s}$ across all conditions. The error bars represent mean \pm standard error of the mean across monkeys 
passage of time seems to have little or no effect on how PI affects the rhesus monkeys' memory performance, despite the large total time differences shown in Table 1. What did affect PI were the trials (i.e., events) that separated the interfering stimulus from the test stimulus. Therefore, in the next experiment we manipulated the nature of these events by using novel stimuli and comparing PI from novel stimuli to that from the familiar stimuli that were used in Experiments 1 and 2 .

\section{Experiment 3}

The results of Exp 1 and 2 suggest that the events of prior trials were functional in producing proactive interference in monkeys, not time separations or time ratios for these events. Therefore, as a test of this apparent "event-based" PI, we manipulated the events themselves by using novel picture stimuli and conducted the same tests as in Experiment 1 to determine whether or not these novel events would change the PI functions.

\section{Method}

\section{Procedure}

For the unfamiliar-stimulus condition, we used a completely novel set of 1,024 picture stimuli that the monkeys had never seen before with the same task parameters as those used in Experiment 1 (six-session blocks with 1-s, 10-s, and 20-s delays each, with an ITI of $15 \mathrm{~s}$ ).

\section{Results and discussion}

The results from this experiment were similar to Experiment 1 in the sense that there was an effect of intervening items, $F(5$, $10)=6.14, p<.01$, no effect of delay, $F(2,4)=1.35, p=.357$, no interaction, $F(10,20)=1.95, p=.081$, and compete overlap of PI functions showing that these time manipulations had no discernable effect on the monkey's PI. Therefore, the results from the three delays in this experiment were averaged to yield a single PI function. This mean PI function is compared to the mean PI function from the three delays used in Experiment 1 in Fig. 4.

Comparing these two mean PI functions revealed a significant main effect of intervening items, $F(5,10)=34.16, p<$ .001. Importantly, there was also a significant interaction between intervening items and stimulus type (unfamiliar vs. familiar) showing that the types of intervening events actually did affect the degree of interference, $F(5,10)=18.12, p<$ .001 , unlike intervening time. PI at $n-1$ and $n-2$ separations was greater for the novel-unfamiliar stimuli than it was for the familiar stimuli, $F(1,2)=29.96, p<.05$, suggesting that

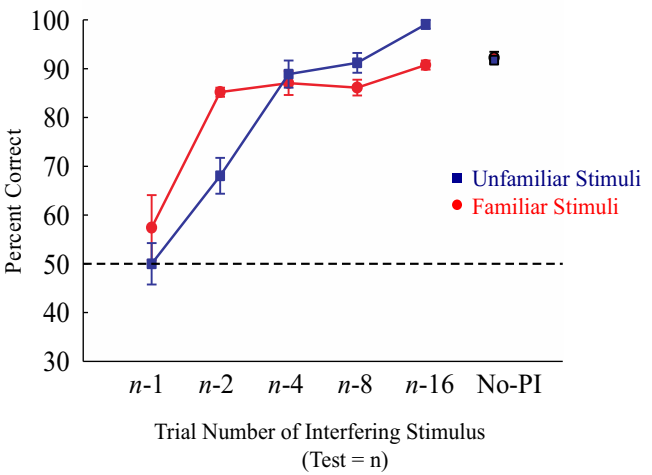

Fig. 4 Mean performance of three monkeys at five interfering-stimulus separations on interference trials (PI trials) compared with baseline performance (No-PI trials) with two different stimulus sets, an unfamiliar stimulus set where the pictures were novel at the beginning of the experiment and a familiar stimulus set used in prior training and experiments. The error bars in Fig. 4 represent mean \pm standard error of the mean across monkeys

encounters with novel stimuli were better remembered and therefore produced more interference than encounters with familiar stimuli. Moreover, these PI functions crossed at $n-4$ and for greater separations ( $n-8$ and $n-16)$ the unfamiliar stimuli actually produced less PI than the familiar stimuli. This result of a transition from greater PI to less PI for better remembered unfamiliar stimuli is somewhat counterintuitive and is most strikingly shown in the $n-16$ condition, where at test, a repeat of the unfamiliar stimulus actually produces enhanced performance instead of interference relative to the nointerference baseline condition, $t(2)=9.12, p=.01$. This "proactive enhancement" effect suggests that monkeys remembered that this novel stimulus had been encountered several trials prior, and not at any other time (i.e., not as the sample stimulus on the test trial); therefore, this stimulus could not have been the sample stimulus on the current trial. Said otherwise, they made the correct "different" response at a somewhat higher rate than when having to rely solely on their memory of the sample stimulus on the current trial.

\section{General Discussion}

The study of intrusions on human short-term or working memory repeatedly surfaces as a topic of considerable interest and debate, apparently because working memory is never completely insulated from the past. Such intrusions from long-term memory have been identified across many visual, auditory, and verbal memory tasks (Crowder, 1976; Hartshorne, 2008; Jacoby et al., 1989; Kane \& Engle, 2000; Keppel \& Underwood, 1962; Makovski \& Jiang, 2008; Postle, Berger, Goldstein, Curtis, \& D'Esposito, 2001; Visscher, Kahana, \& Sekuler, 2009). Similar intrusions have been reported for nonhuman animals (monkeys, chimpanzees, rats, pigeons, and chickadees) in visual and auditory tasks (Bigelow \& Poremba, 2013; Dunnett \& Martel, 1990; Grant, 
1975, 1981; Hampton, Shettleworth, \& Westwood, 1998; Hayes \& Thompson, 1953; Hogan, Edwards, \& Zentall, 1981; Overman \& Doty, 1980; Wright, 2007; Wright et al., 1986, 2012).

Effects of past intrusions on working memory performance have for the most part focused on proactive interference (PI), but have been mostly limited to PI from the immediately preceding trial (Edhouse \& White, 1988; Grant, 1975; Hogan et al., 1981; Makovski \& Jiang, 2008; Reynolds \& Medin, 1981; Worsham, 1975). Likewise, in the experiments of this article, we also found that the effect of PI was strongest at the $n-1$ condition. Although immediately preceding-trial PI effects are important to show, they are relatively limited in terms of understanding the larger process of how proactive interference affects later memory. Here, we systematically manipulated tests of PI to understand how far back in time and across trials, PI affects memory performance. We found with trialunique baseline trials that PI decreased memory performance by as much as $15 \%$, even when a stimulus was repeated from eight trials ago. Importantly, by testing the effects of PI over a considerable range of previous trials, we found that changing delays (Experiment 1) or intertrial intervals (Experiment 2) had little or no effect on the monkey's PI function. Manipulating the delay period and the intertrial interval greatly changed the time between the test stimulus and the interfering stimulus as shown in Table 1. These profound differences in elapsed time had little or no effect on the monkeys' memory performance.

By contrast, in a companion experiment the passage of time greatly affected the pigeon's PI functions (Wright, Katz, \& Ma, 2012). In this companion experiment, we tested pigeons with the same procedures used to test monkeys in Experiment 1 including: same/different procedure, picture stimuli, stimuli visual angles, ITI (15 s), short and moderate testing delays (1 s, $10 \mathrm{~s}$ ), and stimulus-item separations between interference and test stimuli $(n-1, n-2, n-4, n-8, n-16$, No-PI). We also

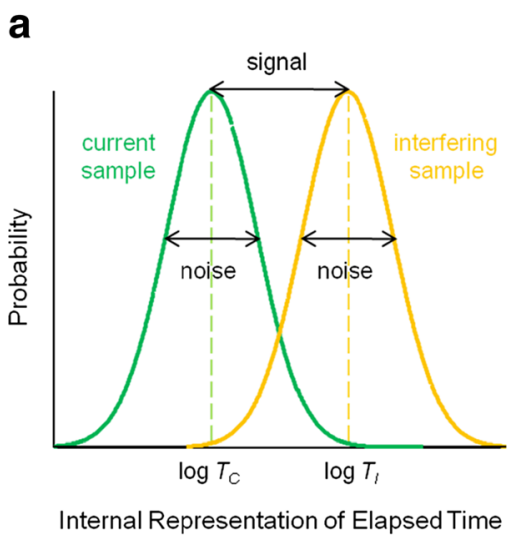

Fig. 5 a Model of the pigeon's decision based on a likelihood ratio from noisy evidence and a criterion for responding "same"/“different" (see text for more details). $\mathbf{b}$ Model fits to the mean performance of four pigeons at conducted the same ANOVA statistical tests showing statistically significant effects (all $p s<.02$ ) for stimulus-item separations, testing delays, and stimulus-item separation $\times$ testing delay interaction. The last two results clearly show that time (delay) had a pronounced effect on the pigeon's PI, and are in stark contrast to the lack of statistically significant effects for time differences (delay or ITI) or interaction for the monkey's PI results shown in this article. These statistically significant results for pigeons were the basis for modeling these results to show how the pigeon's PI does vary with time, unlike the results from the similar tests with monkeys.

This model showed that the pigeon makes its decision (see Fig. 5a) according to the likelihood ratio from noisy evidence and a criterion for responding "same"/"different". Performance is predicted to depend only on the ratio of the time elapsed since the offset of the current sample (i.e., delay time, denoted $T_{\mathrm{C}}$ ) to the time elapsed since the offset of the interfering sample (denoted $T_{\mathrm{I}}$ ), according to the following expression:

$P C_{\text {model }}=\frac{1}{2}+\frac{1}{2}(1-g) \operatorname{erf} \frac{-\log \frac{T_{\mathrm{C}}}{T_{\mathrm{I}}}-b}{2 \sigma}$,

where $P C_{\text {model }}$ is the proportion of correct responses predicted by the model, erf is the error function, $g$ is the guessing rate, $b$ is the response bias, and $\sigma$ is the standard deviation of the noise. The model was fit to the functions (see Fig. 5b) for both the 1-s and the 10-s delay, simultaneously for each subject, thereby providing considerable constraint to each fit. This model provided an excellent quantitative match and accounted for $95 \%$ of the variance $\left(R^{2}=.95\right)$ for the proactiveinterference functions from both delays (simultaneously) and also the No-PI condition. This is the classic Weber-Fechner fraction which accounted for $95 \%$ of the variance and does so

b

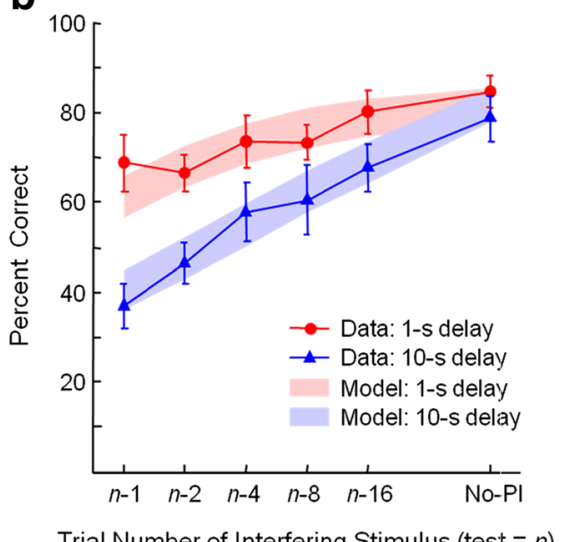

five interfering-stimulus separations on interference trials and mean baseline performance (No-PI trials) across delays of $1 \mathrm{~s}$ and $10 \mathrm{~s}$ (after Wright, Katz, \& Ma, 2012) 
in precisely the way that internal representation of elapsed time has been shown to follow the well-known WeberFechner law or log scale of time (e.g., Buhusi \& Meck, 2005; Gibbon, 1977).

The reason that this study is so relevant to the present article is that if primate PI was similarly affected by time, then the monkey's PI functions should have been similarly affected by time. But there was absolutely no discernable effect of time on the monkey's PI functions, not even when the delay time was twice as long (Experiment 1,20-s delay function) as the longest delay (10 s) used in the pigeon experiment. The monkey's PI functions completely overlapped one another. This lack of any discernable effect of time on the monkey's PI function is astounding (to us at least) in itself, but the contrast between pigeons and monkeys makes it even more remarkable. Nevertheless, all functions from both species showed a pronounced proactive-interference effect. If the monkeys' PI effect is not time based, then the only alternative is that it must be event based. In order to assess this likely possibility, the events themselves were manipulated in Experiment 3 by using unfamiliar stimuli in place of the familiar stimuli, but with the same procedures, delays, and times used in Experiment 1. Just like in Experiment 1, delay time and total time had no effect on the monkeys' PI functions for unfamiliar stimuli. Nevertheless, the mean PI function for unfamiliar items did show greater proactive interference for recently seen "interfering" items, and a performance enhancing effect for items seen more distantly in the past - a proactive enhancing effectrelative to the mean PI function for familiar stimuli. As previously described, both of these effects make perfect sense in terms of event/item based proactive interference.

The effect of interference on memory due to the number of intervening events rather than a mere passage of time has been classically shown in a study with rugby players (Baddeley \& Hitch, 1977). This study showed that the probability of correctly recalling the names of the teams that recently played the games was not affected due to mere decay but rather by the number of intervening games. In that study, recall was tested over many weeks, demonstrating the effects of interference on long-term memory. Other studies have also shown that the representation of stimuli in visual working memory depends on information in long-term memory and is susceptible to the same hallmarks of retrieval from long-term memory (e.g., Bartlett, 1932; Brady, Konkle, \& Alvarez, 2011).

Proactive interference is a fundamental component of memory because most things are seldom encountered in isolation and are preceded by other things. It is unclear why pigeons are so affected by time between events and monkeys are not. Among the possibilities that might bear on this species difference are issues related to explicit as opposed to implicit memory processing. If pigeons primarily depend on familiarity to make these same/different judgments, then that might help explain their dependence on the relative passage of time in terms of confusions due to interference. By contrast, if rhesus monkeys are more explicit processors, then they might be able to bind contexts and better remember the experience of actually seeing an item on a previous trial, resulting in less confusion when this item reappears the test item on the test trial. This seems to be the case, particularly when interfering items were unfamiliar to the monkeys. They apparently use this prior experience and information to bolster their task performance relative their No-PI baseline performance. The primate brain, including frontal and temporal lobe circuits (e.g., Nee, Jonides, \& Berman, 2007; Ranganath, 2010), may be somewhat better equipped than the avian brain for making such inductive judgments, but any such verification will require considerably more research, possibly including primates with lesser (e.g., new world monkeys) or greater (e.g., humans) prefrontal cortical development.

Acknowledgments Support for this research was provided by NIMH Grants R01MH-072616 and R01MH091038 to A. A. W. This research was conducted following the relevant ethics guidelines for research with animals and was approved by UTHSC's institutional IACUC. Requests for reprints and other correspondence may be addressed to: Deepna T. Devkar, Email: deepna.85@gmail.com, or Anthony A. Wright, Department of Neurobiology and Anatomy, University of Texas Medical School at Houston, P.O. Box 20708, Houston, Texas 77225. Email: anthony.a.wright@uth.tmc.edu

\section{References}

Baddeley, A. D., \& Hitch, G. J. (1977). Recency re-examined. In S. Dornic (Ed.), Attention and performance VI (pp. 647-6671). Hillsdale: Erlbaum.

Bartlett, F. C. (1932). Remembering: A study in experimental and social psychology. Cambridge: Cambridge University Press.

Bigelow, J., \& Poremba, A. (2013). Auditory proactive interference in monkeys: The roles of stimulus set size and intertrial interval. Learning \& Behavior, 41(3), 319-332.

Brady, T. F., Konkle, T., \& Alvarez, G. A. (2011). A review of visual memory capacity: Beyond items and toward structured representations. Journal of Vision, 11(5), 4. 1-34.

Buhusi, C. V., \& Meck, W. H. (2005). What makes us tick? Functional and neural mechanisms of interval timing. Nature Reviews Neuroscience, 6(10), 755-765.

Cowan, N. (2005). Working memory capacity. New York: Psychology Press.

Crowder, R. G. (1976). Principles of learning and memory. Hillsdale: Erlbaum.

Dunnett, S. B., \& Martel, F. L. (1990). Proactive-interference effects on short-term-memory in rats: I. Basic parameters and drug effects. Behavioral Neuroscience, 104(5), 655-665.

Edhouse, W. V., \& White, K. G. (1988). Sources of proactive-interference in animal memory. Journal of Experimental Psychology-Animal Behavior Processes, 14(1), 56-70.

Gibbon, J. (1977). Scalar expectancy-theory and Webers Law in animal timing. Psychological Review, 84(3), 279-325.

Grant, D. S. (1975). Proactive interference in pigeon short-term memory. Journal of Experimental Psychology: Animal Behavior Processes, 1(3), 207-220. 
Grant, D. S. (1981). Intertrial interference in rat short-term-memory. Journal of Experimental Psychology-Animal Behavior Processes, 7(3), 217-227.

Hampton, R. R., Shettleworth, S. J., \& Westwood, R. P. (1998). Proactive interference, recency, and associative strength: Comparisons of black-capped chickadees and dark-eyed juncos. Animal Learning \& Behavior, 26(4), 475-485.

Hartshorne, J. K. (2008). Visual working memory capacity and proactive interference. LOS ONE, 3(7).

Hayes, K. J., \& Thompson, R. (1953). Nonspatial delayed response to trial-unique stimuli in sophisticated chimpanzees. Journal of Comparative and Physiological Psychology, 46(6), 498-500.

Hogan, D. E., Edwards, C. A., \& Zentall, T. R. (1981). Delayed matching in the pigeon: Interference produced by the prior delayed matching trial. Animal Learning \& Behavior, 9(3), 395-400.

Jacoby, L. L., Kelley, C., Brown, J., \& Jasechko, J. (1989). Becoming famous overnight: Limits on the ability to avoid unconscious influences of the past. Journal of Personality and Social Psychology, 56(3), 326-338.

Jonides, J., \& Nee, D. E. (2006). Brain mechanisms of proactive interference in working memory. Neuroscience, 139(1), 181-193.

Kane, M. J., \& Engle, R. W. (2000). Working-memory capacity, proactive interference, and divided attention: Limits on long-term memory retrieval. Journal of Experimental Psychology: Learning Memory and Cognition, 26(2), 336-358.

Keppel, G., \& Underwood, B. J. (1962). Proactive inhibition in shortterm retention of single items. Journal of Verbal Learning and Verbal Behavior, 1(3), 153-161.

Makovski, T., \& Jiang, Y. V. (2008). Proactive interference from items previously stored in visual working memory. Memory \& Cognition, $36(1), 43-52$.

Nee, D. E., Jonides, J., \& Berman, M. G. (2007). Neural mechanisms of proactive interference-resolution. NeuroImage, 38(4), 740-751.
Overman, W. H., \& Doty, R. W. (1980). Prolonged visual memory in macaques and man. Neuroscience, 5(11), 1825.

Postle, B. R., Berger, J. S., Goldstein, J. H., Curtis, C. E., \& D’Esposito, M. (2001). Behavioral and neurophysiological correlates of episodic coding, proactive interference, and list length effects in a running span verbal working memory task. Cognitive, Affective, \& Behavioral Neuroscience, 1(1), 10-21.

Ranganath, C. (2010). Binding items and contexts: The cognitive neuroscience of episodic memory. Current Directions in Psychological Science, 19(3), 131-137.

Reynolds, T. J., \& Medin, D. L. (1981). Stimulus interaction and between-trials proactive-interference in monkeys. Journal of Experimental Psychology-Animal Behavior Processes, 7(4), 334347.

Sands, S. F., \& Wright, A. A. (1980). Primate memory: Retention of serial list items by a rhesus-monkey. Science, 209(4459), 938-940.

Standing, L., Conezio, J., \& Haber, R. N. (1970). Perception and memory for pictures: Single-trial learning of 2500 visual stimuli. Psychonomic Science, 19(2), 73-74.

Visscher, K. M., Kahana, M. J., \& Sekuler, R. (2009). Trial-to-trial carryover in auditory short-term memory. Journal of Experimental Psychology-Learning Memory and Cognition, 35(1), 46-56.

Worsham, R. W. (1975). Temporal discrimination factors in delayed matching-to-sample task in monkeys. Animal Learning \& Behavior, 3(2), 93-97.

Wright, A. A. (2007). An experimental analysis of memory processing. Journal of the Experimental Analysis of Behavior, 88(3), 405-433.

Wright, A. A., Katz, J. S., \& Ma, W. J. (2012). How to be proactive about interference: Lessons from animal memory. Psychological Science, 23(5), 453-458.

Wright, A. A., Urcuioli, P. J., \& Sands, S. F. (1986). Proactive interference in animal memory research. In D. F. Kendrick, M. Rilling, \& R. Denny (Eds.), Theories of animal memory (pp. 101-125). Hillsdale: Erlbaum. 